\title{
Study of virulence factors in coagulase-negative staphylococci isolated from newborns
}

\author{
Maria de Lourdes Ribeiro de Souza da Cunha/ ${ }^{+}$, Ligia Maria Suppo de Souza Rugolo*, \\ Carlos Alberto de Magalhães Lopes
}

\begin{abstract}
Departamento de Microbiologia e Imunologia, Instituto de Biociências * Departamento de Pediatria, Faculdade de Medicina, Universidade Estadual Paulista, Unesp, 18618-000 Botucatu, SP, Brasil
\end{abstract}

Coagulase-negative staphylococci (CNS) have been identified as the etiological agent in various infections and are currently the microorganisms most frequently isolated in nosocomial infections. However, little is known about the virulence factors produced by CNS that contribute to the pathogenesis of infections caused by these microorganisms. The study of CNS isolated from infectious processes of newborns hospitalized in the Neonatal Unit of the Hospital of the Botucatu Medical School, Unesp, indicated Staphylococcus epidermidis as the most frequently isolated species (77.8\%), which was also associated with clinically significant situations. The analysis of virulence factors revealed the production of slime in 20 (17.1\%) of all CNS samples isolated and the synthesis of a broad spectrum of enzymes and toxins, including hemolysins (19.6\%), lipase (17.1\%), lecithinase (3.4\%), DNAse (15.4\%), thermonuclease (7.7\%), and enterotoxin A, B or C (37.6\%). Taking into consideration that the etiological importance of CNS has often been neglected, the present investigation confirmed that these microorganisms should not be ignored or classified as mere contaminants.

Key words: coagulase-negative staphylococci - virulence factors - slime - enzymes - toxins

Few reports of infections with coagulase-negative staphylococci (CNS) were published before the 1970s; clinicians and microbiologists considered them to be contaminants of clinical samples, with Staphylococcus aureus being the only pathogenic species within the genus Staphylococcus (Kloos \& Bannerman 1995). This distinction, which has been widely used for clinical diagnosis, represents a challenge in relation to the role of these microorganisms in infectious processes.

At present, CNS are considered to be basically opportunistic microorganisms that prevail in numerous organic conditions, producing serious infections (Kloos \& Bannerman 1995, Lark et al. 2000). The recognition of CNS as etiological agents may also be due to the valorization of this group of organisms as opportunistic pathogens and to the increasing use of invasive procedures such as intravascular catheters and to prosthetic interference. Data obtained between 1995 and 1998 by the Surveillance and Control of Pathogens of Epidemiologic Importance (SCOPE) program have demonstrated that CNS are the etiologic agents most frequently found in nosocomial bacteremias in the United States (Edmond et al. 1999).

The virulence factors produced by CNS and how they contribute to the pathogenicity of infections associated with foreign bodies are currently under investigation. Evidence indicates that pathogenicity might be related to the production of an extracellular polysaccharide, known as slime, that permits these microorganisms to adhere to

\footnotetext{
Financial support: Fapesp

${ }^{+}$Corresponding author: cunhamlr@ibb.unesp.br

Received 19 April 2006

Accepted 12 July 2006
}

smooth plastic surfaces, colonizing catheters, prosthetic heart valves, pacemakers, and joint prostheses (Vogel et al. 2000).

Differentiation between virulent and non-virulent strains has been difficult since the virulence factors of these microorganisms are still not well defined (Gemmell 1987). According to this author and to Koneman et al. (1997), CNS produce other virulence factors, such as hemolysins, lipases, proteases, and toxins.

Based on the above considerations, we decided to evaluate this question in our institution, with the main objectives being the identification of CNS species isolated from clinical cases of newborns hospitalized in the Neonatal Unit of the Hospital of the Botucatu Medical School, Unesp, Botucatu, Brazil, and the determination of the production of slime, enzymes, and toxins by the different isolates.

\section{MATERIALS AND METHODS}

Organisms - CNS isolates were obtained from 107 newborns hospitalized in the Neonatal Unit of the University Teaching Hospital, Unesp, Botucatu, between 1990 and 1996. The procedures used were approved by the Ethics Committee of the School.

Identification of CNS - CNS were isolated on blood agar. Bacterial colonies were stained by the Gram method and submitted to catalase and coagulase tests (Koneman et al. 1997). The genus Staphylococcus was differentiated from Micrococcus by the glucose oxidation and fermentation test and by resistance to bacitracin $(0.04 \mathrm{U})$ and sensitivity to furazolidone $(100 \mu \mathrm{g})$ as described by Koneman et al. (1997).

CNS species were identified as described by Kloos and Schleifer (1975), Kloos and Bannerman (1995), and Cunha et al. (2004). The following tests were used: utiliza- 
tion of xylose, arabinose, sucrose, trehalose, mannitol, maltose, lactose, xylitol, ribose and fructose, characterization of hemolysis, nitrate reduction, urease, decarboxylase ornithine, and resistance to novobiocin.

The following international CNS reference strains were used as controls: S. epidermidis (ATCC 12228), S. simulans (ATCC 27851), S. warneri (ATCC 10209), S. xylosus (ATCC 29979), and S. saprophyticus (ATCC 15305).

Clinical relevance - Data were obtained by analysis of the patients' medical records. Data regarding perinatal infection risk factors were reviewed, including prolonged membrane rupture ( $>24 \mathrm{~h}$ ), gestational age, birthweight, and invasive procedures such as arterial or venous umbilical catheterization, central venous or peripheral catheterization, mechanical ventilation, surgical procedures, peritoneal dialysis, parenteral nutrition, chest drainage, and ventriculoperitoneal shunts. The possibility that foreign bodies had been removed during CNS infection was also examined.

The progression of the clinical situation of the newborn during the week before and after CNS isolation was analyzed, with emphasis on diagnosis and clinical charts suggestive of CNS infection, characterized by insidious and non-specific symptoms, more frequently affecting general health, thermal instability, and apnea (Hall et al. 1987).

With progression of the clinical situation, alterations in the blood count and/or positivity for C-reactive protein were determined at the time of CNS isolation. Normal hematological parameters were those proposed by Manroe et al. (1979). The deaths observed were attributed to CNS infection occurring within the first $72 \mathrm{~h}$ after agent isolation, and possible association with CNS between days 4 and 7 after CNS isolation.

Another aspect investigated and considered to be of clinical relevance was the previous use of antibiotic therapy, including antibiotics adequate for CNS after bacteriological diagnosis, as well as the use of specific antibiotics such as vancomycin, oxacillin, or teicoplanin.

The CNS isolated in the present study were classified as "significant" and "contaminant" according to modified CDC criteria (Garner et al. 1996), as follows:

"Significant" - CNS isolated from newborns who presented three or more of the following features: risk factors for infection, clinical or hematological alterations, and adequate antibiotic therapy. Isolates were also considered to be significant in patients who presented only two of these features and who died without receiving adequate antibiotic therapy.

"Contaminant" - CNS isolated from newborns who presented only risk factors for infection and/or only one of the other features (clinical or hematological alterations, or adequate antibiotic therapy). Isolates from newborns who presented all three features, but showed a satisfactory course of the infection without the administration of adequate antibiotics were also considered to be contaminants. The isolation of another etiological agent from internal fluids and foreign bodies at the time of CNS isolation was also used as a criterion for the classification of contamination.
Study of slime production - Slime production was analyzed as described by Christensen et al. (1982).

Determination of hemolysin production - The production of hemolysins and cytolytic toxins was determined on plates containing blood agar base consisting of 5\% rabbit blood and $5 \%$ sheep blood incubated at $37^{\circ} \mathrm{C}$ for 24 $\mathrm{h}$. A positive result was indicated by the formation of hemolysis zones around the isolated colonies.

Determination of lipase and lecithinase - Lipolytic activity (Jessen et al. 1959) was determined on plates containing blood agar base enriched with $0.01 \% \mathrm{CaCl}_{2} \cdot 2 \mathrm{H}_{2} \mathrm{O}$ and $1 \%$ Tween 80 . A positive result was defined as the formation of opacity around the colonies after incubation at $37^{\circ} \mathrm{C}$ for $18 \mathrm{~h}$, followed by incubation for $24 \mathrm{~h}$ at room temperature. The production of lecithinase (Owens 1974) was studied in Baird-Parker medium. A positive result was indicated by the formation of an opaque halo around the colonies.

Determination of DNAse and TNAse - Nuclease (DNAse) and thermonuclease (TNAse) were determined by the metachromatic Toluidine blue O-DNA agar diffusion technique according to Lachica et al. (1971). Positive results were interpreted by comparing the halos obtained with the $S$. aureus reference strain (ATCC 25923), DNAse, and positive TNAse.

Culture supernatants obtained by the sac culture method (Donnelly et al. 1967), as described below, were also tested for DNAse and TNAse production.

Production of toxins - The toxigenic profile of the isolates was determined using the sac culture method of toxin production (Donnelly et al. 1967). Culture supernatants obtained were stored at $-20^{\circ} \mathrm{C}$ until the time of use.

Detection of enterotoxins and TSST-1 - Enterotoxins and toxic shock syndrome toxin 1 (TSST-1) were detected by the reversed passive latex agglutination (RPLA) assay as described by Shingaki et al. (1981). The SET-RPLAT900 and TST-RPLA-TD940 (Oxoid Diagnostic Reagents) kits were used for the detection of enterotoxin A (SEA), enterotoxin B (SEB), enterotoxin C (SEC) and enterotoxin D (SED), and of TSST-1, respectively. The culture supernatant was previously treated with $5 \%(\mathrm{v} / \mathrm{v})$ normal rabbit serum or $5 \%$ purified rabbit IgG to prevent the occurrence of nonspecific reactions (Pereira et al. 1997). Samples that presented nonspecific reactions even after these procedures were filtered through a Millipore membrane $(8 \mu \mathrm{m})$ and, if necessary, diluted $1: 10$ with $0.02 \mathrm{M}$ phosphate buffer in $0.9 \% \mathrm{NaCl}, \mathrm{pH} 7.4$.

The following international CNS reference strains were used as controls: S. aureus (ATCC 13565, SEA producer), S. aureus (ATCC 14458, SEB producer), S. aureus (ATCC 19095, SEC producer), and S. aureus (ATCC 23235, SED producer).

Statistical analysis - The data were analyzed by the $\chi^{2}$ test or Fisher's exact test, with $\mathrm{n}<20$. The nonparametric Mann-Whitney test was used for the analysis of newborn birthweight and age. The level of significance was set at $\mathrm{p}<0.05$ for all tests. 


\section{RESULTS}

Organisms - A total of 117 CNS were isolated from different materials collected from 107 newborns. Sixty isolates were obtained from blood cultures collected between 1990 and 1996, 41 isolates were from foreign bodies (30 from catheter tips, 10 from cannula tips, 1 from a chest drain tip), 13 from secretions ( 2 drain secretions, 5 gastric secretions, 6 tracheal secretions), and three from urine, all obtained between 1994 and June 1996.

Identification of CNS - S. epidermidis was the CNS species most frequently isolated, corresponding to 91 (77.8\%) isolates. The remaining species were distributed among $S$. haemolyticus (7 isolates, 6\%), S. lugdunensis (7 isolates, 6\%), S. hominis (5 isolates, 4.3\%), S. simulans (4 isolates, 3.4\%), S. warneri (2 isolates, 1.7\%), and $S$. xylosus (1 isolate, $0.8 \%$ ).

Clinical relevance - Of the 60 blood culture isolates, $35(58.3 \%)$ were interpreted to be significant and $25(41.7 \%)$ to be contaminants. Of the $41 \mathrm{CNS}$ isolated from foreign bodies, $21(51.2 \%)$ were considered to be significant, including 14 isolates from catheter tips (66.7\%), six from cannula tips $(28.6 \%)$, and one from a chest drain tip (4.7\%). Of the 13 isolates from secretions, four were considered to be significant, including one from chest drain secretion and three from tracheal secretion.

Of the 107 newborns, 54 had CNS infection and 53 were infection-free. Table I shows that $27(50 \%)$ of the infected newborns presented a birthweight $<1500 \mathrm{~g}$, significantly different from infection-free newborns (20.8\%). Median birthweight also showed a significant difference between the CNS-infected group (1495 g) and the infection-free group $(2270 \mathrm{~g})$. Median age at CNS isolation differed significantly between the infection group (10 days old) and the infection-free group (4 days old). Most CNSinfected newborns $(42,77.8 \%)$ were submitted to two or more invasive procedures, including the use of a catheter in $48(88.9 \%)$, parenteral nutrition in $35(64.8 \%)$, and mechanical ventilation in $33(61.1 \%)$.
The results showed a higher frequency of $S$. epidermidis associated with infection $(86.7 \%)$ than with contamination $(68.4 \%)(\mathrm{p}<0.05)$. No significant differences were observed for the other species.

Clinically significant CNS were isolated at a higher proportion from blood than secretions $(p<0.05)$, but there was no statistically significant difference when compared to foreign bodies.

Slime production - Fig. 1 shows that $20(17.1 \%)$ of the $117 \mathrm{CNS}$ isolates were positive for slime production, including $18.7 \%$ of $S$. epidermidis strains, $28.6 \%$ of $S$. lugdunensis strains, $20 \%$ of $S$. hominis strains. S. haemolyticus, S. simulans, S. warneri or S. xylosus strains were negative for slime production.

Table II shows that $12(23.1 \%)$ of the 52 significant $S$. epidermidis isolates were slime producers compared to five $(12.8 \%)$ of the 39 contaminant isolates, with no significant difference between groups. Slime production was not observed in any of the other significant species. Among contaminant samples, two of the four $S$. lugdunensis samples and one of the five $S$. hominis samples produced slime.

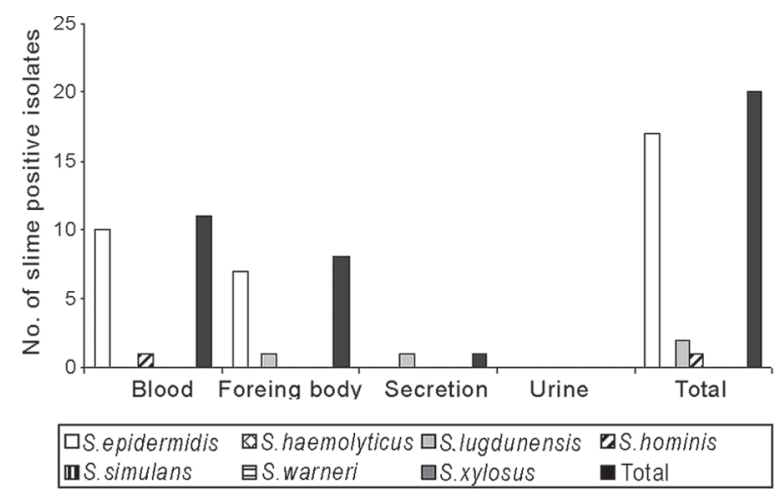

Fig. 1: production of slime by coagulase-negative staphylococci according to species and clinical material. S: Staphylococcus.

TABLE I

Perinatal risk factors of coagulase-negative staphylococci infection

\begin{tabular}{|c|c|c|c|c|c|c|c|}
\hline \multirow[t]{2}{*}{ Risk factors } & \multicolumn{2}{|c|}{ With infection } & \multicolumn{2}{|c|}{ Infection free } & \multicolumn{2}{|c|}{ Total } & \multirow[b]{2}{*}{$\mathrm{p}$ value } \\
\hline & $\mathrm{N}$ & $\%$ & $\mathrm{~N}$ & $\%$ & $\mathrm{~N}$ & $\%$ & \\
\hline Median BW (g) & 1495 & & 2270 & & 1985 & & 0.0011 \\
\hline $\mathrm{BW}<1500 \mathrm{~g}$ & 27 & 50 & 11 & 20.8 & 39 & 36.4 & 0.0002 \\
\hline Median age (days) & 10 & & 4 & & 7 & & 0.0042 \\
\hline Catheter & 48 & 88.9 & 33 & 62.3 & 81 & 75.7 & 0.0004 \\
\hline Chest drainage & 10 & 18.5 & 5 & 9.4 & 15 & 14 & ns \\
\hline Mechanical ventilation & 33 & 61.1 & 18 & 34 & 51 & 47.7 & 0.0138 \\
\hline Parenteral nutrition & 35 & 64.8 & 22 & 41.5 & 57 & 53.3 & 0.0040 \\
\hline Ventriculoperitoneal shunt & 3 & 5.5 & 2 & 3.8 & 5 & 4.7 & ns \\
\hline $\begin{array}{l}\text { Non-removal of } \\
\text { foreign body }\end{array}$ & 31 & 54.7 & 17 & 32.1 & 48 & 44.8 & 0.0010 \\
\hline Membrane rupture $>24 \mathrm{~h}$ & 18 & 33.3 & 17 & 32.1 & 35 & 32.7 & ns \\
\hline Two or more foreign bodies & 42 & 77.8 & 20 & 37.7 & 62 & 57.9 & 0.0001 \\
\hline Total of neonates & 54 & 50.5 & 53 & 49.5 & 107 & 100 & \\
\hline
\end{tabular}

BW: birthweight; ns: $\mathrm{P}>0.05$ (values did not differ significantly at the $5 \%$ level of significance). 
TABLE II

Frequency of enzyme- and slime-producing coagulase-negative staphylococci isolates according to species and clinical relevance

\begin{tabular}{|c|c|c|c|c|c|c|c|c|c|c|c|c|}
\hline \multirow[b]{4}{*}{ Enzymes } & \multicolumn{12}{|c|}{ Species } \\
\hline & \multicolumn{2}{|c|}{ S. epidermidis } & \multicolumn{2}{|c|}{ S. haemolyticus } & \multicolumn{2}{|c|}{ S. lugdunensis } & \multicolumn{2}{|c|}{ S. hominis } & \multicolumn{2}{|c|}{ S. simulans } & \multicolumn{2}{|c|}{ S. warneri } \\
\hline & $\mathrm{S}^{\mathrm{ns}}$ & $\mathrm{C}^{\mathrm{ns}}$ & $\mathrm{S}$ & $\mathrm{C}$ & $\mathrm{S}$ & $\mathrm{C}$ & $\mathrm{S}$ & $\mathrm{C}$ & $\mathrm{S}$ & $\mathrm{C}$ & $\mathrm{S}$ & $\mathrm{C}$ \\
\hline & $52^{a}$ & 39 & 2 & 5 & 3 & 4 & 0 & 5 & 1 & 3 & 1 & 1 \\
\hline Hemolysin & 7 & 4 & 2 & 3 & 1 & 4 & 0 & 0 & 0 & 0 & 1 & 0 \\
\hline Lipase & 6 & 8 & 1 & 1 & 0 & 1 & 0 & 0 & 0 & 2 & 0 & 1 \\
\hline Lecithinase & 1 & 1 & 0 & 1 & 0 & 0 & 0 & 0 & 0 & 0 & 1 & 0 \\
\hline DNAse & 3 & 6 & 1 & 1 & 3 & 2 & 0 & 0 & 0 & 2 & 0 & 0 \\
\hline TNAse & 2 & 3 & 0 & 0 & 1 & 2 & 0 & 0 & 0 & 1 & 0 & 0 \\
\hline Slime & 12 & 5 & 0 & 0 & 0 & 2 & 0 & 1 & 0 & 0 & 0 & 0 \\
\hline
\end{tabular}

S: Staphylococcus; a: total number of strains; S: clinically significant strains ; C: contaminant strains; ns: P >0.05 (values did not differ significantly at the $5 \%$ level of significance).

Enzyme production - The distribution of significant S. epidermidis isolates according to enzyme production is shown in Table II. Enzyme concentration is important for the identification of CNS producers of DNAse and TNAse. Direct detection of DNAse and TNAse in supernatants from overnight cultures in BHI broth only revealed production by one S. lugdunensis strain. However, when the culture supernatants were concentrated by the sac culture method (Donnelly et al. 1967), production was observed in various species.

Of the 52 clinically significant $S$. epidermidis isolates, seven produced hemolysin, six lipase, three DNAse, and two TNAse. No significant difference in enzyme production was observed between the S. epidermidis contaminant isolates and the clinically significant isolates.

The two clinically significant enzyme-producing $S$. haemolyticus isolates produced hemolysin, one produced lipase, and one produced DNAse (Table II). Among the three clinically significant $S$. lugdunensis isolates, one produced hemolysin, all three produced DNAse, and one produced TNAse (Table II).

Production of enterotoxins and TSST-1 - Fig. 2 shows that $44(37.6 \%)$ isolates produced one or a combination of two or more enterotoxins. The distribution of CNS toxin producers according to clinical relevance is shown in Table III. Of the 52 clinically significant $S$. epidermidis isolates, $18(34.6 \%)$ produced enterotoxins, 14 of them producing only SEC, one concomitantly producing SEA and SEB, two producing SEB and SEC, and one simultaneously producing SEA, SEB, and SEC. There was no significant difference in enterotoxin production between clinically significant and contaminant $S$. epidermidis isolates.

Simultaneous production of SEA and SEB and SEB and SEC was also observed in one clinically significant $S$. lugdunensis strain (Table III). Isolated production of SEC was observed in one isolate each of contaminant $S$. haemolyticus, S. lugdunensis, S. hominis, and S. simulans (Table III). The only $S$. simulans strain considered to be significant also produced SEC, whereas $S$. warneri and $S$. xylosus produced no enterotoxins. None of the $117 \mathrm{CNS}$ studied produced SED or TSST-1.

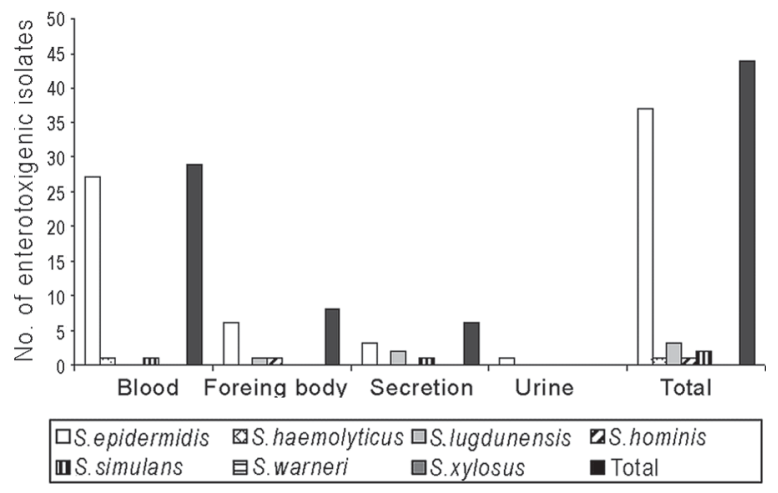

Fig. 2: production of enterotoxins by coagulase-negative staphylococci according to species and clinical material. S: Staphylococcus.

\section{DISCUSSION}

CNS are important microorganisms indigenous to humans and have emerged over recent years as etiologic agents in a series of infections (Hall et al. 1987, Kloos \& Bannerman 1995). In a study carried out by the Centers for Disease Control and Prevention (Atlanta, GA) between 1986 and 1994, involving 99 hospitals, 13,179 cases of infection in newborns were confirmed, with CNS being the agents most frequently isolated in hospital infections (Gaynes et al. 1996). In the 1990s, CNS appeared as the main cause of sepsis in neonatal intensive care units, with an incidence of 33 to $55 \%$ among very low birthweight infants $(<1500 \mathrm{~g})$ (Kacica et al. 1994). The occurrence of CNS in neonatal intensive care units has been attributed to the higher survival rates of premature low birthweight newborns and to procedures such as the use of vascular catheters, parenteral nutrition, mechanical ventilation, and prolonged hospital stays (Fleer \& Verhoef 1984).

In the present study, S. epidermidis was the most frequently isolated species $(77.8 \%)$, in agreement with other investigators (Hall et al. 1987, Neumeister et al. 1995). It was also the most frequently isolated species in clinically significant situations, such as newborns with infection (88.9\%). The predominance of S. epidermidis among CNS 
TABLE III

Frequency of toxin-producing coagulase-negative staphylococci strains according to species and clinical relevance

\begin{tabular}{|c|c|c|c|c|c|c|c|c|c|c|}
\hline \multirow[b]{4}{*}{ Toxins } & \multicolumn{10}{|c|}{ Species } \\
\hline & \multicolumn{2}{|c|}{ S. epidermidis } & \multicolumn{2}{|c|}{ S. haemolyticus } & \multicolumn{2}{|c|}{ S. lugdunensis } & \multicolumn{2}{|c|}{ S. hominis } & \multicolumn{2}{|c|}{ S. simulans } \\
\hline & $\mathrm{S}^{\mathrm{ns}}$ & $\mathrm{C}^{\mathrm{ns}}$ & $\mathrm{S}$ & $\mathrm{C}$ & $\mathrm{S}$ & $\mathrm{C}$ & $\mathrm{S}$ & $\mathrm{C}$ & $\mathrm{S}$ & $\mathrm{C}$ \\
\hline & $52^{a}$ & 39 & 2 & 5 & 3 & 4 & 0 & 5 & 1 & 3 \\
\hline SEA & 0 & 0 & 0 & 0 & 0 & 0 & 0 & 0 & 0 & 0 \\
\hline SEB & 0 & 0 & 0 & 0 & 0 & 0 & 0 & 0 & 0 & 0 \\
\hline SEC & 14 & 17 & 0 & 1 & 0 & 1 & 0 & 1 & 1 & 1 \\
\hline SED & 0 & 0 & 0 & 0 & 0 & 0 & 0 & 0 & 0 & 0 \\
\hline TSST-1 & 0 & 0 & 0 & 0 & 0 & 0 & 0 & 0 & 0 & 0 \\
\hline $\mathrm{SEA}+\mathrm{SEB}$ & 1 & 0 & 0 & 0 & 1 & 0 & 0 & 0 & 0 & 0 \\
\hline $\mathrm{SEB}+\mathrm{SEC}$ & 2 & 2 & 0 & 0 & 1 & 0 & 0 & 0 & 0 & 0 \\
\hline $\mathrm{SEA}+\mathrm{SEB}+\mathrm{SEC}$ & 1 & 0 & 0 & 0 & 0 & 0 & 0 & 0 & 0 & 0 \\
\hline Total & $18^{\mathrm{ns}}$ & $19^{\text {ns }}$ & 0 & 1 & 2 & 1 & 0 & 1 & 1 & 1 \\
\hline
\end{tabular}

S: Staphylococcus; $a$ : total number of strains; S: clinically significant strains; C: contaminant strains; SEA: enterotoxin A; SEB: enterotoxin B; SEC: enterotoxin C; SED: enterotoxin D; TSST-1: toxic shock syndrome toxin-1; ns: P $>0.05$ (values did not differ significantly at the $5 \%$ level of significance).

species causing infection in newborns has been recognized by other investigators, with its frequency ranging from 60 to $90 \%$ (Hall et al. 1987, Neumeister et al. 1995).

Although $S$. epidermidis is the most frequent etiological agent, other pathogenic CNS species have been isolated from various clinical sources (Kloos \& Bannerman 1995). In the present study, other species were found to be associated with infection, including two S. haemolyticus strains, three $S$. lugdunensis strains, and one strain each of S. simulans, S. warneri, and S. xylosus. Hall et al. (1987) isolated three $S$. haemolyticus strains, two $S$. hominis strains, two $S$. warneri strains, and one $S$. simulans strain from children with clinical and laboratory evidence of sepsis and also pneumonia.

There are few reports of infection with $S$. lugdunensis in newborns, probably because this species has only been described recently (Freney et al. 1988). However, studies on adult patients have shown that this species is an opportunistic and significant pathogen (Fleurette et al. 1989, Lambe et al. 1990).

The mechanisms by which CNS provoke infections have not been completely elucidated. However, in opportunistic situations, these microorganisms cross protection barriers such as the skin and mucosa and colonize sites adjacent to the normal flora. Several authors have emphasized the production of exopolysaccharide or slime as an epidemiological marker of infection (Christensen et al. 1982, Hall et al. 1987, Vogel 2000). On the other hand, other investigators have found no association between slime-producing strains and the occurrence of infections caused by these microorganisms (Christensen et al. 1983, Riley \& Schneider 1992).

The present study shows that a small proportion of the isolates which produce this exopolysaccharide (22.2\%) are associated with infectious processes. Other authors also found no evidence of slime being a virulence factor (Christensen et al. 1982). Riley and Schneider (1992) also suggested that slime production does not seem to be an important virulence factor of $S$. saprophyticus isolated from women with urinary tract infection.

Various experiments using animal models have been carried out to determine the importance of slime as a virulence factor. Baddour et al. (1984), in an experimental study on catheter-induced endocarditis in rats, observed a difference in virulence between $S$. epidermidis and $S$. hominis. All animals inoculated with $S$. epidermidis developed endocarditis compared to only $12.5 \%$ of animals inoculated with $S$. hominis $(\mathrm{p}<0.001)$. However, the authors found no association between slime production and the development of endocarditis, suggesting that this polysaccharide is not a critical determinant of virulence. Additionally, Patrick et al. (1992), using mice with subcutaneous implants, observed that slime-producing samples did not increase the risk of infection. The authors suggested that traumatized tissue, associated with the presence of catheters, might be a sufficient condition for the development of infections caused by CNS, and that factors other than slime-mediated colonization determine the pathogenicity of these microorganisms.

However, if slime production promotes adherence to prostheses, thus acting as a virulence factor, infection control becomes more difficult since it protects CNS cells from antimicrobial agents and the host's natural defense mechanisms. Gray et al. (1984) reported that the mucous substance produced by CNS can interfere with the cellmediated immune response. Davenport et al. (1986) showed that only $32 \%$ of infections caused by slime-producing CNS were cured by antibiotics, whereas a $100 \%$ success rate was achieved for non-producing strains. These results suggest that the control of infections caused by slime-producing CNS requires the removal of the prosthesis, as well as conventional antibiotic therapy.

Slime production can also vary among different species. According to Christensen et al. (1983), this characteristic is more frequent in strains of $S$. capitis, $S$. epidermidis, S. hominis, and S. saprophyticus. Among 
the CNS species isolated in the present study, slime production was observed in S. epidermidis, S. lugdunensis, and $S$. hominis. Slime production by S. lugdunensis has also been reported by Fleurette et al. (1989).

The study of CNS pathogenicity has also shown that various metabolites are produced by these microorganisms, including enzymes and toxins which may play a role in the pathogenicity of these microorganisms (Gemmell 1987). Other authors have observed the production of hemolysins or cytolytic toxins by CNS. In the present study, hemolysins were produced by isolates of S. epidermidis, S. haemolyticus, S. lugdunensis, and S. warneri, but not by strains of $S$. hominis, $S$. simulans, and $S$. xylosus. Similar results have been reported by Kloos and Schleifer (1975), Fleurette et al. (1989), Lambe et al. (1990), and Cunha et al. (2004).

The production of lipase, DNAse, and TNAse by these organisms has also been reported. Lambe et al. (1990) verified that most $S$. epidermidis, $S$. warneri, and $S$. hominis strains included in their study produced lipase and DNAse. In the present investigation, except for $S$. hominis and S. xylosus, all species produced lipase. With regard to DNAse and TNAse production, production of these enzymes was observed in strains of S. epidermidis, S. lugdunensis, and S. simulans, and production of DNAse by $S$. haemolyticus. Production of TNAse by $S$. lugdunensis has also been demonstrated by Fleurette et al. (1989). Gramoli and Wilkinson (1978) detected TNAse production in some strains of S. xylosus, S. simulans, S. capitis, and S. sciuri.

Among the species included in the present study, $S$. epidermidis was the only one that produced all of these exoenzymes, but when the isolates involved in the etiology of the infections were compared with the contaminant isolates, no significant difference was observed. These findings are similar to those reported by Nataro et al. (1994), and suggest that the infections caused by these microorganisms do not only depend on virulence factors but also on the conditions that predispose the host to infection, including factors innate to newborns and the use of invasive procedures. Analysis of risk factors in newborns indicated that a birthweight $<1500 \mathrm{~g}$, the presence of foreign bodies - catheters, mechanical ventilation, parenteral nutrition etc. - and non-removal of foreign bodies were factors significantly predisposing to CNS infection.

In the present study, concomitant production of SEA + SEB and SEB + SEC and isolated production of SEC were observed in these organisms. Production of SEC was also detected in one $S$. haemolyticus strain, one $S$. hominis strain, and one S. simulans strain, but production of SED and TSST-1 was not observed in any of the CNS studied.

Valle et al. (1991) found a toxigenic capacity in 45 $(16.5 \%) \mathrm{CNS}$ isolated from goats, including S. epidermidis, S. haemolyticus, S. warneri, and S. xylosus, which simultaneously produced TSST- 1 and TSST-1 + SEC. Crass and Bergdoll (1986) reported the isolated production of SEA and SEC, or in combination with TSST-1, by CNS isolated from patients with toxic shock syndrome or other infections. However, production of TSST-1 by CNS has been questioned by other investigators who did not confirm these findings (Parsonnet et al. 1987, Kreiswirth et al. 1987).

Although much controversy about the production of these toxins by CNS still exists, the present results and those reported by other authors demonstrate that their toxic ability cannot be ignored. According to Bergdoll and Chesney (1991), these microorganisms have been associated with the etiology of serious staphylococci infections, and there is no reason not to consider them as toxic.

The exact role of extracellular staphylococci products in the pathogenicity of a systemic infection is still unclear. Enterotoxins and TSST-1 have received renewed attention by researchers due to their "superantigen" properties. As "superantigens", enterotoxins bind directly to the class II major histocompatibility complex, without the typical process of normal antigens, resulting in the stimulation of many $\mathrm{T}$ cells and, therefore, an overproduction of cytokines such as interleukin-1 (IL-1), IL-2, gamma interferon, and tumor necrosis factor alpha (Marrack \& Kappler 1990). Current evidence indicates that the physiological events that occur in neonatal sepsis are mediated by activated cytokines in response to the presence of bacterial components (Kilpatrick \& Harris 1998). The fact that these toxins are superantigens and that the release of immunological mediators increases the inflammatory response observed during the pathogenesis of sepsis and neonatal shock suggests that enterotoxins and TSST-1 may play an important role in the progression of infections caused by toxigenic CNS strains.

The existing divergences regarding the toxigenicity of CNS emphasize the need for further studies using sensitive and reliable genotypic techniques to confirm the ability of these staphylococci to produce toxins.

Our study revealed the presence of one or more virulence factors in $77.8 \%$ of the CNS strains isolated, suggesting that CNS virulence factors provide a selective advantage for skin colonization of hospitalized newborns. Very low birthweight newborns submitted to invasive procedures show a higher risk of subsequent infection with these strains.

\section{REFERENCES}

Baddour LM, Christensen GD, Bisno AL 1984. Production of experimental endocarditis by coagulase-negative staphylococci: variability in species virulence. J Infect Dis 150: 721727.

Bergdoll MS, Chesney PJ 1991. Toxic Shock Syndrome, CRC Press, Boca Raton, 234 pp.

Christensen GD, Parisi JT, Bisno AL, Simpson WA, Beachey EH 1983. Characterization of clinically significant strains of coagulase-negative staphylococci. J Clin Microbiol 18: 258-269.

Christensen GD, Simpson WA, Bisno AL, Beachey EH 1982. Adherence of slime-producing strains of Staphylococcus epidermidis to smooth surfaces. Infect Immun 37: 318-326.

Crass BA, Bergdoll MS 1986. Involvement of coagulase-negative staphylococci in toxic shock syndrome. J Clin Microbiol 23: $43-45$. 
Cunha MLRS, Sinzato YK, Silveira LVA 2004. Comparison of methods for the identification of coagulase-negative Staphylococcus. Mem Inst Oswaldo Cruz 99: 855-860.

Davenport DS, Masanari RM, Pfaller MA, Bale MJ, Hierholzer WJ 1986. Usefulness of a test for slime production as a marker for clinically significant infections with coagulasenegative staphylococci. J Infect Dis 153: 332-339.

Donnelly CB, Leslie JE, Black LA, Lewis KH 1967. Serological identification of enterotoxigenic staphylococci from cheese. Appl Microbiol 15: 1382-1387.

Edmond MB, Wallace SE, McClish DK, Pfaller MA, Jones RN, Wenzel RP 1999. Nosocomial bloodstream infections in United States hospitals: a three-year analysis. Clin Infect Dis 29: 239-244.

Fleer A, Verhoef J 1984. New aspects of staphylococcal infections: emergence of coagulase-negative staphylococci as pathogens. Antonie Leeuwenhoek 50: 729-744.

Fleurette J, Bes M, Brun Y, Freney J, Forey F, Coulet M, Reverdy ME, Etienne J 1989. Clinical isolates of Staphylococcus lugdunensis and S. schleiferi: bacteriological characteristics and susceptibility to antimicrobial agents. Res Microbiol 140: 107-118.

Freney J, Brun Y, Bes M, Meugnier H, Grimont F, Grimont PAD, Nervi C, Fleurette J 1988. Staphylococcus lugdunensis sp. nov. and Staphylococcus schleiferi sp. nov., two species from human clinical specimens. Int J Syst Bacteriol 38: 168172.

Garner JS, Jarvis WR, Emori TG, Horan TC, Hughes JM 1996. $\mathrm{CDC}$ definitions for nosocomial infections. In RN Olmsted, APIC Infection Control and Applied Epidemiology: Principles and Practice, Mosby, St. Louis, p. A1-A20.

Gaynes RP, Edwards JR, Jarvis WR, Culver DH, Tolson JS, Martone WJ 1996. Nosocomial infections among neonates in high-risk nurseries in the United States. Pediatrics 98: 357-361.

Gemmell CG 1987. Exo-proteins of coagulase-negative staphylococci as possible virulence factors. Zentralb Bakteriol Mikrobiol Hyg Abt 116 (Suppl.): 93-102.

Gramoli JL, Wilkinson BJ 1978. Characterization and identification of coagulase-negative heat-stable deoxyribonucleasepositive staphylococci. J Gen Microbiol 105: 275-285.

Gray ED, Peters G, Verstegen M, Regelmenn WE 1984. Effect of extracellular slime substance from Staphylococcus epidermidis on the human cellular immune response. Lancet 1:365-367.

Hall RT, Hall SL, Barnes WG, Izuegbu J, Rogolsky M, Zorbas I 1987. Characteristics of coagulase-negative staphylococci from infants with bacteremias. Pediatr Infect Dis J 6: 377383.

Jessen O, Faber V, Rosendal K, Eriksen KR 1959. Some properties of Staphylococcus aureus, possibly related to pathogenicity. I. A study of 446 strains from different types of human infection. Acta Pathol Microbiol Scand 47: 316-326.

Kacica MA, Horgan MJ, Ochoa L, Sandler R, Lepow ML, Venezia R 1994. Prevention of gram-positive sepsis in neonates weighing less than 1500 grams. J Pediatr 125: 253258.

Kilpatrick L, Harris MC 1998. Cytokines and the inflammatory response. In RA Polin, WW Fox (eds), Fetal and Neo- natal Physiology, WB Saunders, Philadelphia, p. 1967-1989.

Kloos WE, Bannerman TL 1995. Staphylococcus and Micrococcus. In PR Murray, EJ Baron, MA Pfaller, FC Tenover, RH Yolken (eds), Manual of Clinical Microbiology, American Society Microbiology, Washington, p. 282-298.

Kloos WE, Schleifer KH 1975. Simplified scheme for routine identification of human Staphylococcus species. J Clin Microbiol 1: 82-88.

Koneman EW, Allen SD, Janda WM, Schreckenberger PC, Winn Jr WC 1997. Color Atlas and Textbook of Diagnostic Microbiology, JB Lippincott, Philadelphia, 1395 pp.

Kreiswirth BN, Schlievert PM, Novick RP 1987. Evaluation of coagulase-negative staphylococci for ability to produce toxic shock syndrome toxin 1. J Clin Microbiol 25: 2028-2029.

Lachica RUF, Genigeorgis C, Hoeprich PD 1971. Metachromatic agar-diffusion methods for detecting staphylococcal nuclease activity. Appl Microbiol 21: 585-587.

Lambe Jr DW, Ferguson KP, Keplinger JL, Gemmell CG, Kalbfleisch JH 1990. Pathogenicity of Staphylococcus lugdunensis, Staphylococcus schleiferi, and three other coagulase-negative staphylococci in a mouse model and possible virulence factors. Can J Microbiol 36: 455-463.

Lark RL, Chenoweth C, Saint S, Zemencuk JK, Lipsky BA, Plorde JJ 2000. Four year prospective evaluation of nosocomial bacteremia: epidemiology, microbiology, and patient outcome. Diagn Microbiol Infect Dis 38: 131-140.

Marrack P, Kappler J 1990. The staphylococcal enterotoxins and their relatives. Science 248: 705-717.

Manroe BL, Weinberg AG, Rosenfeld CR, Browne R 1979. The neonatal blood count in health and disease. I. Reference values for neutrophilic cells. J Pediatr 95: 89-98.

Nataro JP, Corcoran L, Zirin S, Swink S, Taichman N, Goin J, Harris MC 1994. Prospective analysis of coagulase-negative staphylococcal infection in hospitalized infants. $J$ Pediatr 125: 798-804.

Neumeister B, Kastner S, Conrad S, Klotz G, Bartmann P 1995. Characterization of coagulase-negative staphylococci causing nosocomial infections in preterm infants. Eur J Clin Microbiol Infect Dis 14: 856-863.

Owens JJ 1974. The egg yolk reaction produced by several species of bacteria. J Appl Bacteriol 37: 137-148.

Parsonnet J, Harrison AE, Spencer SE, Reading A, Parsonnet KC, Kass EH 1987. Nonproduction of toxic shock syndrome toxin 1 by coagulase-negative staphylococci. J Clin Microbiol 25: 1370-1372.

Patrick CC, Plaunt MR, Hetherington SV, May SM 1992. Role of the Staphylococcus epidermidis slime layer in experimental tunnel tract infections. Infect Immun 60: 1363-1367.

Pereira ML, Heneine LGD, Pereira JL, Bergdoll MS 1997. Control of nonspecific reactions on reversed passive latex agglutination assay (RPLA) for detecting nanogram quantities of staphylococcal enterotoxins. Arq Bras Med Vet Zootec 49: 493-497.

Riley TV, Schneider PF 1992. Infrequency of slime production by urinary isolates of Staphylococcus saprophyticus. J Infect 24: 63-66.

Shingaki M, Igarashi H, Fujikawa H, Ushioda H, Terayama T, 
Sakai S 1981. Study on reversed passive latex agglutination for the detection of staphylococcal enterotoxins A-C. Annu Rep Tokyo Metr Res Lab Publ Hlth 32: 128-131.

Valle J, Vadillo S, Piriz S, Gomez-Lucia E 1991. Toxic Shock Syndrome Toxin 1 (TSST-1) production by staphylococci isolated from goats and presence of specific antibodies to
TSST-1 in serum and milk. Appl Environ Microbiol 57: 889891.

Vogel L, Jacobus HS, Spaargaren J, Suiker I, Dijkshoorn L 2000. Biofilm production by Staphylococcus epidermidis isolates associated with catheter related bacteremia. Diagn Microbiol Infect Dis 30: 138-141. 